\title{
VI. Nachspiel: Rechtliche Konkretisierung und verfassungsgerichtliche Überprüfung des BSHG 1962-1967
}

Wichtiges Ziel der Fürsorgereformer war die bundesweite Vereinheitlichung des Fürsorgerechts, nicht zuletzt im verfassungsmäßig begründeten Interesse „der Einheitlichkeit der Lebensverhältnisse über das Gebiet eines Landes hinaus“ (Art. 72 Abs. 2 GG). ${ }^{1}$ Dementsprechend ließ das BSHG den Ländern sehr viel geringere Spielräume zur eigenen Rechtssetzung als seine Weimarer Vorgänger. ${ }^{2}$ Die 1962/63 erlassenen neuen Ausführungsgesetze der Länder regelten fast ausschließlich organisatorische und finanzielle Fragen wie die Heranziehung kreisangehöriger Gemeinden, Zuständigkeit von Behörden und überörtlichen Trägern, Kostenverteilung, Antragsverfahren etc. ${ }^{3}$ Nur zum Teil griffen sie dabei auf einen von einer Arbeitsgruppe der Länderreferenten 1961 mit dem Bundesinnenministerium erstellten Musterentwurf zurück ${ }^{4}$, so daß Besonderheiten der Landesverfassung, aber auch unterschiedliche politische Ziele des Landesgesetzgebers, etwa bei der institutionalisierten Zusammenarbeit mit der freien Wohlfahrtspflege 5

1 Vgl. auch Wehlitz, Bundessozialhilfegesetz, S. 64.

2 Mit Inkrafttreten des BSHG am 1.6.1962 wurden die bisherigen Ausführungsgesetze der Länder gegenstandslos.

3 Für die Fundstellen der Länderausführungsgesetze (in Hamburg erging nur eine Anordnung zur Durchführung des BSHG) in ihrer zeitgenössischen Fassung siehe Gottschick, Bundessozialhilfegesetz, 1966, S. 9f.

4 Bereits vor der Zustimmung des Bundesrates hatte diese Gruppe ihre Arbeit aufgenommen und unter dem 31.8.1961 einen Musterentwurf vorgelegt; vgl. Vermerk Referat V 4 vom 2.5.1961; die Ergebnisniederschriften über die Sitzungen des Unterausschusses der Leitenden Fürsorgereferenten der Länder [...] am 24./25.5., 15./16.6., 13./14.7. und 30./31. 8.1961; Musterentwurf eines Landesausführungsgesetzes zum Bundessozialhilfegesetz, 31.8.1961; Auszug aus dem Kurzprotokoll über die Tagung der Leitenden Fürsorgereferenten am 20./21.9.1961, BAK, B 106-9697. Deutlich anders als der Musterentwurf waren vor allem die Regelungen von Bayern, Berlin und Hamburg.

5 Die Arbeitsgruppe der Länderreferenten hatte es für grundsätzlich „nicht tunlich“ gehalten, weitere landesrechtliche Bestimmungen über die Beteiligung der freien Verbände vorzusehen, „da diese geeignet sein können, die Praxis zu erschweren“, Ergebnisniederschrift über die Sitzung des Unterausschusses der Leitenden Fürsorgereferenten am 24./25.5.1961, BAK, B 106-9697. Ausnahmen seien nur dort zu erwägen, wo schon bisher gesetzliche Regelungen über die Zusammenarbeit bestanden. Entsprechend sah Bayern auch weiterhin eine Beteiligung der freien Wohlfahrtspflege und der Kirchen in den landesüblichen Sozialausschüssen und Arbeitsgemeinschaften, vgl. Art. 2ff., 6, 14 Gesetz zur Ausführung des Bundessozialhilfegesetzes vom 26.10.1962, Bayerisches GVBl. S.272, Baden-Württemberg ebenfalls Arbeitsgemeinschaften, vgl. $\int 7$ Gesetz zur Ausführung des Bundessozialhilfegesetzes vom 23.4.1963, Gesetzblatt für Baden-Württemberg S. 33, Hessen eine Mitwirkung der freien Verbände im dortigen Landesbeirat für Sozialhilfe, vgl. $\ 18$ Hessisches Ausführungsgesetz zum Bundessozialhilfegesetz vom 28.5. 1962, GVBl. für das Land Hessen Teil I S.273, vor. Einzig Rheinland-Pfalz regelte 
oder der Zuständigkeit für die Festsetzung der Regelsätze ${ }^{6}$, weiterhin zum Tragen kamen.

Diese Beschränkung auf formalrechtliche Regelungen bedeutete jedoch nicht, daß die Länder keinen Einfluß auf die weitere materielle Ausgestaltung des BSHG besaßen und besitzen. Vielmehr ermächtigt das BSHG (bzw. das neue SGB XII) zu einer Reihe von Durchführungsverordnungen, die nur mit Zustimmung des Bundesrates erlassen werden können und die z.T. ganz erhebliche Auswirkungen auf die reale Höhe der Leistungen und den Empfängerkreis haben: Das galt zunächst etwa für die Verordnungen zur Eingliederungshilfe für Behinderte, zur Definition des Einkommensbegriffs für die Einkommensgrenzen ${ }^{8}$ oder zur Festlegung der zu schonenden kleineren Vermögensbarbeträge ${ }^{9}$, vor allem aber für die konkrete Ausgestaltung der Regelsätze. Diese, so prophezeite Kurt Wehlitz bereits 1961, werde „für eine nicht geringe Anzahl von Hilfeempfängern möglicherweise weitergehende finanzielle Auswirkungen haben als die in den gesetzlichen Leistungstatbeständen des BSHG vom Gesetzgeber normierten Leistungsverbesserungen“. ${ }^{10}$

Wie Mitte der fünfziger Jahre war es das noch unangefochtene Expertengremium des DV, das wichtige Vorarbeiten leistete. ${ }^{11}$ Bereits seit Mai 1960 beschäftigte sich der personell fast unveränderte Arbeitskreis „Aufbau der Richtsätze“ mit der Aktualisierung des ersten Warenkorbs, der sich als unrealistisch knapp kalkuliert erwiesen hatte, den mittlerweile geänderten Verbrauchergewohnheiten nicht mehr entsprach und nun an der Meßlatte des "menschenwürdigen Lebens“ zu messen war. Tatsächlich erweiterte der Arbeitskreis den Warenkorb um neue Elemente vor allem bei Ernährung und den sogenannten sonstigen persönlichen Bedürfnissen des täglichen Lebens, kalkulierte auch die Zuschläge etwas günstiger und definierte die Relationen der Regelsätze für Haushaltsangehörige zum EckRegelsatz neu. ${ }^{12}$ Die Bedeutung dieses erneuerten Warenkorbes war gar nicht zu

darüber hinaus in Anlehnung an das JWG auch die Unterstützung von Einrichtungen der freien Wohlfahrtspflege noch genauer, vgl. $\mathbb{9}$ Landesgesetz zur Ausführung des Bundessozialhilfegesetzes vom 8.3.1963, GVBl. für Rheinland-Pfalz S. 79.

6 In den meisten Ländern wurden seitdem die Regelsätze von den obersten Landesbehörden festgesetzt; in Nordrhein-Westfalen und Rheinland-Pfalz jedoch gab die oberste Landesbehörde Rahmensätze, in Bayern Mindestsätze vor.

7 Vgl. VO nach $\ 47$ des Bundessozialhilfegesetzes (Eingliederungshilfe-Verordnung) vom 27.5.1964, BGBl. I S.339, die den Kreis der Hilfeberechtigten sowie die gesetzlichen Hilfsmaßnahmen ausführlich definierte; ferner VO zur Durchführung des $\mathbb{8} 81$ Abs. 1 Nr. 1 des Bundessozialhilfegesetzes vom 20.7.1962, BGBl. I S.513, die bestimmte, welche ambulanten Leistungen der stationären Eingliederungshilfe gleichzusetzen und daher mit der besonderen Einkommensgrenze zu veranschlagen waren; VO zur Durchführung des $\ 81$ Abs. 1 Nr. 3 vom 27.5.1964, BGBl. I S.343, wo die ebenfalls unter die besondere Einkommensgrenze fallenden „größeren“ Hilfsmittel definiert wurden.

${ }^{8}$ VO zur Durchführung des $\ 76$ des Bundessozialhilfegesetzes vom 28.11.1962, BGBl. I S. 692 .

${ }_{9}$ VO zur Durchführung des $\ 88$ Abs. 2 Nr. 8 des Bundessozialhilfegesetzes vom 20.7. 1962, BGBl. I S. 514.

10 Wehlitz, Bundessozialhilfegesetz, S. 64.

11 Vgl. Heisig, Armenpolitik, 1995, S.175ff.; Giese, Regelsatzsystem, S. $521 \mathrm{ff}$.

12 Vgl. den Bericht von Käthe Petersen vom Arbeitskreis vor den Leitenden Fürsorgereferenten der Länder am 20./21.9.1961, Auszug aus Kurzprotokoll, BAK, B 106-9697; NDV 42 (1962), S. 59ff., 367ff.; Giese, Regelsatzsystem, S. 521. 
überschätzen: Schließlich würden die auf der Basis des Warenkorbes definierten Regelsätze nicht nur Empfängerkreis und Leistungshöhe bei der Hilfe zum Lebensunterhalt bestimmen, sondern über die an den Regelsatz gekoppelten Einkommensgrenzen auch den Kreis der Empfänger der Hilfe in besonderen Lebenslagen festlegen. ${ }^{13}$ Entsprechend heftig waren die Proteste von seiten der Landkreise gegen den neuen Warenkorb, der viele auf dem Land völlig untypische Bedarfselemente enthalte und zudem die Relation zu niedrigen Arbeitseinkommen nicht berücksichtige. ${ }^{14}$ Tatsächlich sollte die Umsetzung des Warenkorbs den Eck-Regelsatz drastisch von rund 65 DM auf rund 104 DM erhöhen. ${ }^{15}$ Doch die schließlich nach Stellungnahme der kommunalen Spitzenverbände unter Zustimmung des Bundesrates am 20.Juli 1962 erlassene sogenannte Regelsatzverordnung ${ }^{16}$ übernahm zwar die Vorgaben des Arbeitskreises und hatte im Gegensatz zu den Verwaltungsvorschriften von 1955 Gesetzeskraft; sie ließ aber den für die Festsetzung der Regelsätze zuständigen Landesbehörden bzw. kommunalen Trägern noch Spielräume. Laut dieser Verordnung umfaßte der Regelsatz die laufenden Leistungen für Ernährung und Kochfeuerung, für die Beschaffung von kleineren Wäschestücken und Haushaltsgegenständen, für geringe Instandhaltungskosten von Wäsche, Kleidung und Schuhen, sowie für Körperpflege, Beleuchtung, Reinigung und persönliche Bedürfnisse des täglichen Lebens. Der etwas umstrukturierte Eck-Regelsatz galt jetzt auch für Alleinstehende, daneben gab es nun vier Altersgruppen von Haushaltsangehörigen. Bei der generellen Festsetzung der Regelsätze war zwar das ortsübliche Arbeitseinkommen unterer Lohngruppen zu berücksichtigen (Abstandsgebot), im Einzelfall aber der notwendige Lebensunterhalt zu sichern und damit eine Auffanggrenze nicht erlaubt. ${ }^{17}$

Tatsächlich wurde der Warenkorb aber nie flächendeckend in allen Ländern der Bundesrepublik umgesetzt ${ }^{18}$; nichtsdestoweniger bewirkte er zusammen mit der Pflicht zur Anpassung an die Preisentwicklung ( $\$ 22$ Abs. 3 BSHG), daß der EckRegelsatz von 93 DM im Jahre 1961 im Bundesdurchschnitt auf 107 DM ein Jahr später und bis 1970 auf 155 DM stieg. ${ }^{19}$ In der Literatur wird die Regelsatzverordnung in Verbindung mit dem BSHG daher als „Kumulations- und Schlußpunkt einer fürsorgerechtlichen Entwicklung hin zu Standardisierung und Verrechtlichung", als Statuierung eines erstmals national verbindlichen und bedarfsorientierten Existenzminimums gewürdigt ${ }^{20}$, aber auch als problematische Statuierung von

13 Nicht zu reden von der Bedeutung der Regelsätze als Vergleichsmaßstab etwa im Recht der Jugendhilfe, der Kriegsopferversorgung, der Ausbildungsförderung oder im Steuerrecht.

14 Vgl. Heisig, Armenpolitik, 1995, S. $176 f$.

15 Vgl. Giese, Regelsatzsystem, S. 522.

16 Verordnung zur Durchführung des $\ 22$ des Bundessozialhilfegesetzes vom 20.7.1961, BGBl. I S. 515.

17 Zur weiteren Entwicklung und Verschärfung des „Abstandsgebots“, das 1982 schließlich in $\$ 22$ Abs. 3 inkorporiert wurde, vgl. Peter Klein, Regelsatzentwicklung.

18 Vgl. Heisig, Armenpolitik, 1995, S. 184f., 199; auch Giese, Regelsatzsystem, S. 522.

19 Vgl. die Tabelle bei Curtze, Fürsorgeunterstützung, S. 129.

20 Heisig, Armenpolitik, 1995, S. 199; vgl. ferner Leibfried/Hansen/Heisig, Sozialpolitik, S. 64; Rudloff, Fürsorge, S. 198; auch Galperin, Sozialhilfe; Giese, Regelsatzsystem, S. 520. 
einer „Art Mindestlohn“ gewertet. ${ }^{21}$ Zunehmend in die Kritik gerieten allerdings die unzureichende Fortschreibung der Preise und Bedarfselemente ${ }^{22}$, in jüngerer Zeit auch die ihr zugrundeliegende Vorstellungen von Normalfamilien und Normalerwerbsformen, nicht zuletzt schließlich die nicht nur von Zeitgenossen durchaus positiv bewertete „Entpolitisierung" der Festsetzung des Warenkorbs ${ }^{23}$, die dem demokratisch nicht legitimierten DV lange Zeit dominierenden Einfluß auf diese zentrale Bemessungsgröße deutscher Sozialstaatlichkeit zuwies, zumal auch die vermeintliche Wertfreiheit seiner Expertenurteile einer genaueren Prüfung nicht standhielt. ${ }^{24}$ Nichtsdestoweniger konnte das Warenkorb-Modell, das 1970 abermals überarbeitet wurde, knapp zwanzig Jahre lang cum grano salis dem Anspruch zur materiellen Gewährleistung eines „menschenwürdigen Lebens“ genügen. Seitdem allerdings Anfang der achtziger Jahre aufgrund zunehmender Finanzierungsprobleme vor allem kommunaler Protest eine erneute Aktualisierung des Warenkorbs verhinderte, wurde unter Sparvorgaben erst mit verschiedenen neuen Bemessungs-Modellen (Deckelung des Warenkorbs, Statistikmodell u.ä.) experimentiert, seit 1998 dann auf der Basis einer alle fünf Jahre durchgeführten Einkommens- und Verbrauchsstichprobe gearbeitet ${ }^{25}$; seither blieben die Regelsätze hinter der allgemeinen Entwicklung des Lebensstandards zurück. ${ }^{26}$

Während so seit Herbst 1961 die Umstellung auf die neuen Wohlfahrtsgesetze in den Sozialverwaltungen der Länder einsetzte und auf dem Fürsorgetag von 1965 eine erste umfassende Bilanz dieser Gesetzesimplementierung gezogen werden konnte, blieb ein Bereich gleichsam im Wartestand ${ }^{27}$ : die künftige Gestaltung des Verhältnisses von kommunalen Sozialhilfeträgern und den freien Wohlfahrtsverbänden. Während sämtliche Bundestagsparteien nach den weltanschaulichen Gefechten des Wahlkampfes wieder zur politischen Tagesordnung übergingen ${ }^{28}$,

21 Manfred G. Schmidt, Sozialpolitik, S. 87, deutet diese Neuerung des BSHG neben der Dynamisierung der Renten als „Zweite gefährliche Zeitbombe“ für Wirtschaft und Staatshaushalt, die ihre Wirkung dann in konjunkturell ungünstigen Zeiten entfaltete.

22 Vgl. Giese, Regelsatzsystem, S. $520 \mathrm{ff}$.

23 Die negativen Folgen einer stärkeren „Politisierung“ der Festsetzungsmechanismen des Regelsatzes seit Anfang der achtziger Jahre, die nicht mit „Parlamentarisierung“ gleichzusetzen ist, beschreiben Jaedicke u.a., Politik, S. 81ff.

24 Vgl. Hofmann/Leibfried, Regelmäßigkeiten, S. 262ff.; Stolleis, Rechtsgrundlagen, insbesondere S. $102 \mathrm{f}$.

25 Vgl. Jaedicke u.a., Politik, S. 81ff., 151ff.

26 Vgl. Schellhorn, Einordnung, S.170; Rudloff, Fürsorge, S.199; zur Problematik der Gegenüberstellung von Regelsätzen mit Netto-Löhnen und Eckrenten siehe Peter Klein, Regelsatzentwicklung, S. 91f.

$27 \mathrm{Vgl}$. Matthes, Konzeptionen, S. $126 \mathrm{f}$.

28 Anders als zunächst überlegt, verzichtete auch die SPD-Fraktion darauf, die seinerzeitigen Änderungsanträge der FDP nun selbst einzubringen und dadurch die Liberalen in Zugzwang zu bringen; statt dessen sprach sich Willy Brandt anläßlich der Debatte über die Regierungserklärung der neuen Bundesregierung im Bundestag am 6.12.1961 nur allgemein dafür aus, „einige gemeindefeindliche Tendenzen, die in einer Anzahl von Bundesgesetzen Eingang gefunden haben, durch eine sorgfältige Neuberatung zu beseitigen“, BT, 4. Wp. 1961, Sten. Ber., Bd.50, S.61. Bei der ersten Novellierung des BSHG 1965 spielte die Frage der Stellung der freien Verbände aber keine Rolle mehr; vgl. Sitzung des Bundestags am 23.6.1965, BT, 4. Wp. 1961, Sten. Ber., Bd. 59, S. $3596 f$. 
erhoben die sozialdemokratisch regierten Städte Dortmund, Darmstadt, Frankfurt am Main und Herne im Februar 1962 in Karlsruhe Verfassungsbeschwerde gegen die einschlägigen Regelungen des BSHG und des JWG. ${ }^{29}$ Fünf Monate später folgte das ebenfalls sozialdemokratisch regierte Land Hessen mit einer noch weitergehenden Normenkontrollklage, die von Bremen, Hamburg und Niedersachsen unterstützt wurde. ${ }^{30}$

Daraufhin bildete die Subsidiaritätsfrage noch auf Jahre in den betroffenen Ministerien sowie in den kommunalen Spitzenverbänden und den freien Wohlfahrtsverbänden, in den Kirchen und bei Juristen ein mit erheblichem publizistischen Aufwand beackertes Feld der Auseinandersetzung: In umfangreichen Rechtsgutachten, Materialsammlungen und Memoranden, in ersten juristischen Dissertationen und soziologischen Monographien, in zahllosen Abhandlungen in Fachzeitschriften und offiziellen Stellungnahmen wurden auf allen Seiten die bekannten Argumente ausgetauscht, und allenfalls die nun noch stärker offensichtliche innerkirchliche Differenzierung auf evangelischer Seite gab der Debatte neue Akzente. ${ }^{31}$

Nach mehr als fünf Jahren, am 18. Juli 1967, erklärte der Zweite Senat des Bundesverfassungsgerichts mit 4:3 Stimmen die angefochtenen Bestimmungen zur freien Wohlfahrtspflege im BSHG und JWG für mit dem Grundgesetz vereinbar ${ }^{32}$ : Aus dem Sozialstaatsgebot folge keineswegs, daß der Gesetzgeber für die Verwirklichung des Verfassungsziels einer „gerechten Sozialordnung“ nur behördliche Maßnahmen vorsehen dürfe, sondern er könne dafür auch die Mithilfe privater Wohlfahrtsorganisationen festlegen. Mit der Voraussetzung dieser prinzi-

29 Vgl. BVerfGE 22, S. 184ff.; die Verfassungsbeschwerden der Stadt Dortmund gegen die \$S 10 und 93 BSHG sowie gegen das JWG, jeweils vom 21.2.1962, sind abgedruckt in: NDV 42 (1962), S. 120-126. Als Prozeßbevollmächtigte fungierten führende Politiker der Bundestagsparteien: Adolf Arndt (SPD), Thomas Dehler (FDP) und bemerkenswerterweise auch Kurt Sieveking (CDU).

30 Vgl. BVerfGE 22, S. 184ff.; die Anträge der Regierung des Landes Hessen auf Feststellung der Nichtigkeit von Vorschriften des BSHG bzw. des JWG vom 10.7.1962 sind ebenfalls abgedruckt in: NDV 42 (1962), S.325-337. Die Normenkontrollklagen richteten sich auch gegen die Zulassung der Freiheitsentziehung für Gefährdete sowie gegen eine bundesrechtliche Festlegung der örtlichen Träger und die Einbeziehung der Jugendpflege.

31 Vgl. - mit zahlreichen weiterführenden Literaturangaben zur zeitgenössischen Auseinandersetzung - Emmelius, Rangverhältnis; Matthes, Konzeptionen, S. 14ff.; Desch, Subsidiaritätsprinzip, insbesondere S. 182ff.; Brenner, Diakonie, S. 78ff.; Referat V 4 an Referat I A 3, 23.8.1962, BAK, B 106-20024; DCV und Innere Mission/Hilfswerk an Bundeskanzler Ludwig Erhard, 17.3.1965, Durchschrift, ADW, Allg. Slg. B93.1 II. Ferner Stellungnahmen der Bundesvereinigung der Kommunalen Spitzenverbände zur Verfassungsbeschwerde der Städte Dortmund u.a. zum JWG bzw. BSHG, abgedruckt in: NDV 43 (1963), S.114-124; Oel, Hilfe; Hasenclever, Grundlagen; Stellungnahme des DPW zur Verfassungsbeschwerde gegen das BSHG, in: DPWV-Nachrichten 1963, S. 58-60; Caritas (1962); Franz Klein, Staat. Für die evangelische Seite insbesondere Georg Suhr, Hg. Stimmen; Rendtorff, Subsidiaritätsprinzip; Collmer, Hg., Beiträge, einschließlich einer Übersicht über die Interpretationen in einschlägigen damaligen Rechtskommentaren, S.267ff.; Süddeutsche Zeitung vom 5./7.1.1962; Collmer an Ohl, 20.6. 1962, ADW, HGSt, SP-S XXV 1: 427-1/1; Philippi, Subsidiaritätsprinzip.

32 Vgl. BVerfGE 22, S. 180ff.; BGBl. I S. 896. 
piellen Offenheit des Grundgesetzes widersprach das Gericht sowohl der Interpretation der Antragsteller, aber auch der Kleinschen Auslegung. Ansonsten argumentierte das Gericht vor allem historisch: Wie ihre Weimarer Vorläufer gingen BSHG und JWG davon aus, daß Sozial- und Jugendhilfe zwar eine Aufgabe des Staates seien, dieser aber weder organisatorisch noch finanziell diese Aufgabe allein bewältigen könne; dazu bedürfe es vielmehr der gemeinsamen Bemühung von Staat und freien Verbänden: „Diese hergebrachte und durch Jahrzehnte bewährte Zusammenarbeit von Staat und freien Verbänden soll durch die Vorschriften [des BSHG und JWG] gefördert und gefestigt werden. “33 Das Gericht stellte dabei klar, die Bestimmungen verfolgten "nicht den Zweck, der freien Wohlfahrtspflege schlechthin einen Vorrang vor der öffentlichen Sozialhilfe einzuräumen, sondern sie wollen die längst auch im Fürsorgewesen übliche und bewährte Zusammenarbeit [...] gewährleisten, um mit dem koordinierten Einsatz öffentlicher und privater Mittel den größtmöglichen Erfolg zu erzielen". ${ }^{34}$ Mit dieser pragmatischen Argumentation, die auf die Frage der konkreten Ausgestaltung des Verhältnisses von kommunalen Trägern und freien Verbänden nicht näher einging, konnten letztlich alle Seiten leben. ${ }^{35}$ Ohnehin hatte das Urteil zum Zeitpunkt seiner Verkündung schon manches an Brisanz verloren, denn die Zusammenarbeit von öffentlichen Trägern und freien Verbänden funktionierte offensichtlich vielerorts ${ }^{36}$, wenn auch der DV-Vorstand sich noch zu einer Mahnung zur Kooperation veranlaßt sah. ${ }^{37}$

Auf die Frage nach den Konsequenzen der Regelungen für die Entwicklung der freien Wohlfahrtsverbände in der Bundesrepublik gibt es eine Reihe von Antworten: Zweifellos bildeten diese Regelungen durch die rechtliche Absicherung des Status der freien Verbände eine wichtige Voraussetzung für die beispiellose Expansion der freien, insbesondere der konfessionellen Wohlfahrtspflege seit den sechziger Jahren. So stieg allein die Zahl der Betriebe der Caritas von gut $7000 \mathrm{im}$ Jahre 1960 auf fast 18700 im Jahre 2000, die Zahl der dort Vollbeschäftigten in der gleichen Zeit von knapp 100000 auf mehr als $500000 .{ }^{38}$ Allerdings ging dieser $\mathrm{Zu}$ wachs anders, als es das Schlagwort von der „Funktionssperre“ nahelegt, nicht nur auf Kosten der öffentlichen Träger, wie selbst Kritiker vermerken. So stieg der Anteil der Einrichtungen in öffentlicher Trägerschaft an allen Einrichtungen in den ersten zwanzig Jahren nach Inkrafttreten der beiden Wohlfahrtsgesetze von gut 19\% (1961) auf mehr als 27\% (1982), der Anteil an verfügbaren Plätzen im

33 BVerfGE 22, S. 200.

34 Ebenda, S. 202. Für nichtig erklärte das Verfassungsgericht hingegen einen Teil der in der Normenkontrollklage der Länder angefochtenen Organisationsvorschriften (u.a. $\ 96$ Abs. 1 Satz 2 BSHG) sowie u.a. die Bestimmungen zur Freiheitsentziehung Gefährdeter ( $\$ 73$ Abs. 2 u. 3 BSHG); vgl. ebenda, S. 211, 220.

35 So einhellig im Rückblick die einstigen Kontrahenten Könen, Weg, S.411, und Franz Klein, Beitrag, S. 226; aus Sicht der AWO auch Hasenclever, Jugendhilfe, S. 204.

36 Vgl. Hasenclever, Jugendhilfe, ebenda; Schellhorn, Wohlfahrtspflege, S. 78; Osten, Jugend- und Gefährdetenfürsorge, 2002, S. 131ff.

37 Vgl. eine Erklärung des DV-Vorstands vom 12.10.1967 zum Urteil des Bundesverfassungsgerichts, auszugsweise abgedruckt in: Orthbandt, Deutscher Verein, S. 410ff.

382000 einschließlich neue Bundesländer; vgl. Schmitz-Elsen, Gesetzgebung, S. 163. 
selben Zeitraum sogar von rund 19\% auf 30\%. ${ }^{39}$ Überdies profitiert die öffentliche Hand nicht unerheblich von den Ressourcen der freien Verbände, die sonst von öffentlichen Trägern zu übernehmende Leistungen nach wie vor häufig kostengünstiger erbringen und eigene Mittel zuschießen. ${ }^{40}$

Damit wird deutlich, daß sich die Expansion der freien Wohlfahrtsverbände nicht allein den Subsidiaritäts-Regelungen verdankt, sondern gleichfalls der Ausweitung der öffentlichen Wohlfahrtsaufgaben und deren finanzieller Sicherung durch das BSHG und das JWG sowie, vor allem, der enormen Expansion des Gesundheitswesens mit dem bedeutenden Segment der Krankenhäuser in konfessioneller Trägerschaft. Insofern ist die freie Wohlfahrtspflege mehr denn je integraler Bestandteil des bundesrepublikanischen Sozialstaats und ist es auch nach der Wiedervereinigung geblieben: Mitte der neunziger Jahre wurden in Deutschland über zwei Drittel aller Jugendhilfeeinrichtungen, fast 55\% aller Alten- und Behindertenheime sowie jedes dritte Krankenhaus von freien Verbänden getragen. ${ }^{41}$

Die Konsequenzen dieser Einbindung in die sozialstaatliche Aufgabenerfüllung sind dabei vielfach so, wie von den Gegnern der damaligen Regelungen für die freien Verbände prognostiziert: starke finanzielle Abhängigkeit von öffentlichen Mitteln; öffentliche Kontrolle durch Anerkennungs- und Eignungskriterien als Vorbedingung für finanzielle Förderung und damit ein Verlust an Gestaltungsspielräumen; damit verbundene zunehmende Standardisierung des Angebots der freien Verbände, Bürokratisierung ihrer Strukturen und somit die unterschiedlich eingeschätzte Gefahr eines Verlusts an eigenem Profil der freien Träger, das ja gerade eine der Hauptbegründungen für die Subsidiaritäts-Regelungen gewesen war. ${ }^{42}$ Mit den einst so entscheidenden Größen des „Vorrangs“ bzw. „Nachrangs“ jedenfalls läßt sich das Verhältnis von öffentlicher Sozialhilfe und freier Wohlfahrtspflege kaum mehr charakterisieren, an ihre Stelle trat zumindest bis in die achtziger Jahre hinein eine funktionierende Zusammenarbeit, die - je nach Perspektive - zu beschreiben wäre als neo-korporatistischer „komplexer Gesamtverbund“43 und „Kooperationszusammenhang, der durch gegenseitige Abhängigkeiten und Verflechtungen zusammengehalten wird“44, als „enge Verflechtung“45,

39 Vgl. Sachße, Bedeutung, S. 37.

40 So insbesondere die Argumentation von Batkiewicz/Speckert, Finanzen; nach einer Berechnung von Spiegelhalter, Wohlfahrtspflege, entlastete die Tätigkeit allein der Caritas die öffentliche Hand 1990 um 11 Mrd. DM.

41 Vgl. Backhaus-Maul/Olk, Subsidiarität, S. 112.

42 Entsprechend kritisch die Einschätzung der Entwicklung seit 1961 bei Sachße, Bedeutung, S.37f.; Foss, Diakonie, S. 87ff.; Manderscheid, Verflechtung; Backhaus-Maul/Olk, Subsidiarität; Zacher, Grundlagen, S. 515f.; vgl. auch die einschlägigen Beiträge in den Sammelbänden von Sachße, Hg., Wohlfahrtsverbände, sowie Rauschenbach/Sachße/Olk, Hg., Wertgemeinschaft; die gängige These von der geradlinigen Entwicklung speziell der Spitzenverbände der freien Wohlfahrtspflege zu "Großkonzernen“ des Sozialsystems durch die örtliche Perspektive relativierend dort Manderscheid, Wohlfahrtspflege, S.230ff.; deutlich positiver schließlich die Einschätzung der Gesamtentwicklung bei Schellhorn, Wohlfahrtspflege; Kuper, 25 Jahre.

43 Sachße, Bedeutung, S. 38; Sachße, Subsidiarität, 1994, S. 732.

44 Backhaus-Maul/Olk, Subsidiarität, S. 108.

45 Vgl. Manderscheid, Verflechtung, S. 64. 
problematisches „Mischregime“46, „undurchsichtige, informelle Politikverflechtung“ der freien Verbände ${ }^{47}$, „latente Verstaatlichung der Tätigkeit freier Trä-

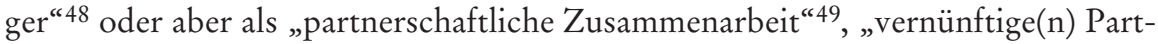

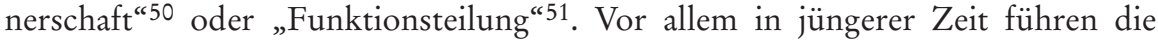
knappen öffentlichen Mittel sogar zu einer überraschenden historischen Pointe, wenn die freien Träger gar nicht mehr alle ihnen angebotenen Aufgaben übernehmen können und selbst das notorisch im öffentlichen sozialen Sektor engagierte Berlin die Hälfte der städtischen Kindertagesstätten in freie Trägerschaft überführen wollte. ${ }^{52}$ Nicht die Vorrang-Regelungen des BSHG, sondern vor allem der öffentliche Spardruck führt nun immer häufiger dazu, daß nach gegenseitiger Absprache der Verbände untereinander und mit den öffentlichen Trägern die einst gefürchteten regionalen Angebotsmonopole tatsächlich entstehen und das diese Regelungen begründende Wahlrecht des Hilfeempfängers untergraben. ${ }^{53}$ Neben diesen finanziellen Problemen und daraus resultierenden Effizienzvorgaben für die öffentlichen Verwaltungen sind es die verstärkte Förderung von verbandsunabhängiger Selbsthilfe (etwa im Kinder- und Jugendhilfegesetz von 1990) ${ }^{54}$, vor allem aber die Öffnung ganzer sozialer Dienstleistungsbereiche für privatgewerbliche Anbieter - insbesondere nach Einführung der Pflegeversicherung und einer entsprechenden Neufassung von $\$ 93$ BSHG 1993/94 und 1996 $55^{55}$, die „eine

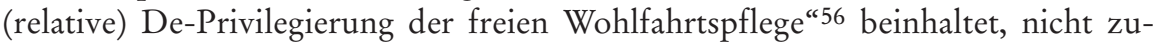
letzt der europäische Einigungsprozeß (der eine Rücksichtnahme auf die rechtliche Sonderstellung der deutschen Spitzenverbände der freien Wohlfahrtspflege kaum erwarten läßt), die eine Ökonomisierung des Wohlfahrtssystems vorantreiben $^{57}$, den traditionellen Dualismus des deutschen Wohlfahrtsstaates aufbrechen und die freien Verbände „im Übergang vom Subsidiaritätsprinzip zum Wettbewerbsprinzip “58 vor ganz neue Herausforderungen stellen. ${ }^{59}$

46 Zacher, Grundlagen, S. 515.

47 Manderscheid, Wohlfahrtspflege, S. 236.

48 Seeber, Caritas, S. 192.

${ }^{49}$ Schmitz-Elsen, Gesetzgebung, S. 58; ähnlich Orthbandt, Deutscher Verein, S. 412; Franz Klein, Beitrag, S. 227; Schellhorn, Wohlfahrtspflege.

50 Könen, Weg, S. 410; so auch Honecker, Sozialethik, S. 631; Kuper, 25 Jahre, S. 60.

51 So, unter Berufung auf Collmer, Brenner, Diakonie, S. 87.

$52 \mathrm{Vgl}$. den damaligen Berliner Stadtentwicklungssenator und Landesvorsitzenden der SPD, Peter Strieder, in einem Interview des Tagesspiegels vom 2.11.2002; für entsprechende Entwicklungen in der Stadt Frankfurt/M. in den neunziger Jahren vgl. Manderscheid, Wohlfahrtspflege, S. 246; allgemein Seeber, Caritas, S. 189; Kuper, 25 Jahre, S. 61.

53 Vgl. Manderscheid, Wohlfahrtspflege, S. 242ff.

54 Vgl. Backhaus-Maul/Olk, Subsidiarität, S. $123 \mathrm{ff}$.

${ }^{55}$ Vgl. ebenda, S. 126ff.; Schellhorn, Bundessozialhilfegesetz, 2002, S. 12, $711 \mathrm{ff}$.

56 Hammerschmidt/Uhlendorff, Hg., Wohlfahrtsverbände, Einleitung, S. 9.

57 Vgl. Rudloff, Fürsorge, S. $226 f$.

${ }^{58}$ Hammerschmidt/Uhlendorff, Hg., Wohlfahrtsverbände, Einleitung, S. 9.

59 Vgl. Olk, Korporatismus; Manderscheid, Wohlfahrtspflege, S. 243ff., sowie insgesamt den Sammelband von Hammerschmidt/Uhlendorff, Hg., Wohlfahrtsverbände. 